\title{
Unmanned Aerial Vehicles Enabled IoT Platform for Disaster Management
}

\author{
Waleed Ejaz 1,*D, Muhammad Awais Azam ${ }^{2}$, Salman Saadat ${ }^{3}$, Farkhund Iqbal ${ }^{4}$ \\ and Abdul Hanan 5 iD \\ 1 Department of Applied Science \& Engineering, Thompson Rivers University, Kamloops, \\ BC V2C 0C8, Canada \\ 2 Faculty of Telecom and Information Engineering, University of Engineering and Technology, Taxila, \\ Punjab 47050, Pakistan \\ 3 Systems Engineering Department, Military Technological College, Muscat 111, Oman \\ 4 College of Technological Innovation, Zayed University, Abu Dhabi 144534, UAE \\ 5 KUST (SE) Department, University of Management and Technology, Sialkot Campus, Sialkot, \\ Punjab 51310, Pakistan \\ * Correspondence: waleed.ejaz@ieee.org
}

Received: 17 June 2019; Accepted: 11 July 2019; Published: 15 July 2019

\begin{abstract}
Efficient and reliable systems are required to detect and monitor disasters such as wildfires as well as to notify the people in the disaster-affected areas. Internet of Things (IoT) is the key paradigm that can address the multitude problems related to disaster management. In addition, an unmanned aerial vehicles (UAVs)-enabled IoT platform connected via cellular network can further enhance the robustness of the disaster management system. The UAV-enabled IoT platform is based on three main research areas: (i) ground IoT network; (ii) communication technologies for ground and aerial connectivity; and (iii) data analytics. In this paper, we provide a holistic view of a UAVs-enabled IoT platform which can provide ubiquitous connectivity to both aerial and ground users in challenging environments such as wildfire management. We then highlight key challenges for the design of an efficient and reliable IoT platform. We detail a case study targeting the design of an efficient ground IoT network that can detect and monitor fire and send notifications to people using named data networking (NDN) architecture. The use of NDN architecture in a sensor network for IoT integrates pull-based communication to enable reliable and efficient message dissemination in the network and to notify the users as soon as possible in case of disastrous situations. The results of the case study show the enormous impact on the performance of IoT platform for wildfire management. Lastly, we draw the conclusion and outline future research directions in this field.
\end{abstract}

Keywords: Internet of Things; 5G and beyond networks; named data networks; wildfire management

\section{Introduction}

The forests and wild-land in British Columbia (BC) cover more than 94 million hectares and they are the most diverse in Canada. There are approximately 8000 wildfires on average each year throughout Canada and an average of 2000 wildfires only in BC [1]. In the aftermath of disasters such as wildfires, information dissemination to and from citizens and authorities often involves the use of mobile network services to seek help, distribute critical information, and possibly to confirm the safety. The traffic on the cellular network typically increases substantially because of disaster. Therefore, efficient and reliable systems are required that can detect and monitor the fire as well as send notifications to the users as soon as possible.

The Internet of Things (IoT) is an important paradigm that has opened a promising door towards catering to multitude of problems (such as transportation, health, disaster management, 
etc.) in an efficient and reliable way [2]. IoT devices are equipped with the ability to sense, process, and communicate to enable communication among physical objects [3]. IoT aims to offer optimal connectivity that can be used in monitoring, controlling and automation of reliable access of users. Recently, IoT based platforms are considered for the disaster management [4]. In context of wildfire disaster, it is extremely important to detect the wildfire and its exact location, as well as early notification to the fire units and users is essential. An efficient IoT platform for wildfire management is expected to lead to major social, economic, and environmental impacts in society. However, IoT platform for disaster management needs memory efficient, robust, and reliable communication among IoT devices.

Further, unmanned aerial vehicles (UAVs) are recently investigated as flying wireless communication platforms [5]. UAVs can be used as relays to extend the coverage and improve connectivity of ground IoT network. On the contrary, UAVs can also be used as base station to provide connectivity to ground IoT network [6]. Compared to the conventional cellular network, UAVs can provide connectivity in areas where the infrastructure is damaged or there is no existing communication infrastructure. Therefore, UAVs can provide solution to collect data from the ground IoT network in the area with limited or no infrastructure. This integration of UAVs and ground IoT network can enhance the ability to efficiently predict, access and respond to wildfire. It will also significantly reduce the response time during disaster management resulting in saving the lives of people in the affected areas [5].

The rules and regulations for UAVs are different depending on the country. However, special permissions are generally granted for the use of UAVs to help first responders in disaster situations. A UAV-enabled IoT platform can work as an effective tool for wildfire services to improve wildfire management at reduced cost. However, it is important to involve all the stakeholders including the government and people to determine the requirements for fire management. BC wildfire services has spent approximately $\$ 568$ million in 2017 [7]. Therefore, it is important to investigate the UAV-enabled IoT platform for wildfire management. There are several strategies proposed in the literature for UAV-enabled disaster management. In a pre-disaster phase, when the conventional network is fully operational, UAVs are mainly deployed to collect data from ground IoT network and acquire data using onboard sensors such as aerial footage and imagery for remote surveillance. For example, UAVs are deployed for real-time mountain fire monitoring in [8]. In [9], images of disaster-hit area are taken using UAVs for image stitching. Moreover, UAVs are deployed to locate survivors [10]. In [11], UAVs are used to collect data from sensors and to forward it to the cloud for further processing in real time. During and post disaster, where a part of traditional network infrastructure is damaged, UAVs can also serve as a permanent data relay. The authors of [12] considered UAV mounted base stations to serve as data relay between IoT devices. In [13-15], UAV-based small cells are used to fill the coverage holes in a cellular network in a post-disaster situation. In disaster scenarios, where the permanent connectivity is not required or is not possible due to the limited number of UAVs and their restricted coverage, UAVs are employed for delay tolerant data collection. In [16], UAV based mobile base station is considered that serves user along its path in post-disaster communication. In [17], UAVs are deployed to collect data from IoT devices on ground and the optimal trajectory of UAVs is studied to minimize energy consumption to enhance their flight time.

The conventional Internet protocol (IP)-based network architecture for ground IoT network has several loopholes in terms of caching, mobility, security, and flexibility [18]. Moreover, the current IP infrastructure is not equipped to deal with disaster situations adequately, especially when the network is fragmented due to damage to the communication infrastructure. Therefore, an efficient and reliable system is needed for ground IoT network in such situations to detect and monitor the fire as well as send notifications to the users. Recently, named data networking (NDN) architecture has received much attention which evolves from the content-centric networking [19]. NDN is a data-oriented future Internet architecture (which retrieves data based on named content) offering several advantages over transmission control protocol (TCP)/IP networking including content delivery efficiency and data security $[20,21]$. Forwarding strategy is the key feature of NDN to realize dynamic, adaptive and intelligent forwarding. Moreover, NDN architecture in ground IoT network can reduce the overall 
delay between IoT nodes, which increases the robustness of the system. Therefore, it is important to develop ground IoT network based on NDN architecture that can efficiently handle remote monitoring.

\section{Contributions}

In this paper, we present an overview of UAV-enabled IoT platform for disaster management. The following are the main contributions of this paper:

- We present a framework for research in UAV-enabled IoT platform for disaster management such as wildfire management.

- We highlight critical hurdles in the success of UAV-enabled IoT platform for disaster management.

- We discuss the use of NDN architecture to enhance the performance of the ground IoT network.

- We provide a case study to show the significance of NDN architecture in the design of efficient ground IoT network that can detect and monitor wildfire and send notifications to people in the affected areas.

- We highlight future research directions for UAV-enabled IoT platform for disaster management.

The the remainder of the paper is organized as follows: Section 2 briefly reviews the literature on UAV-enabled IoT platform for disaster management and applications of UAV-enabled IoT systems in disaster management. The architecture and key challenges are presented in Section 3. In Section 4, we present a case study to use NDN architecture for IoT Platform to increase its performance. Finally, the conclusion is drawn and future research directions are highlighted in Section 5.

\section{Literature Review and Applications in Disaster Management}

In this section, we provide a brief literature review of UAV-enabled IoT platform followed by its applications in disaster management.

\subsection{Literature Review}

The vision of fully autonomous UAV-enabled IoT platform is very challenging mainly due to the need for coordination of UAVs, limited energy, spectrum resources, and technological law enforcement issues. There have been research efforts in recent years for the development of UAVs-enabled IoT platform for various applications. Connectivity and coverage of cellular network serving both UAVs and ground users are major focus areas in this field. A multi-layer UAVs hierarchical architecture is proposed in [22] to integrate UAVs in 5G and beyond networks and various design trade-offs are discussed. For cellular connectivity of low altitude UAVs, the performance of long-term evolution (LTE) connectivity is enhanced while providing a shield to ground users in [23]. In the context of coverage probability, an in-depth analysis is presented in [24] to show the effectiveness of cellular network for providing connectivity to both aerial and ground users. The feasibility of supporting UAVs with existing cellular infrastructure is also studied by researchers, where it is concluded that the interference can be minimized by careful design of network parameters to satisfy the quality of service (QoS) requirements [25]. The density-aware UAV placement is studied in [26] for distributed users such that the number of users served by the UAV-based base station is maximized under the given minimum rate requirement per user. Spectrum is another focus area for cellular connected UAVs. Multicell cooperation, nonorthogonal multiple access (NOMA), and cognitive radio (CR) are promising technologies for future cellular connected UAVs [27-30]. Moreover, a spectrum sensing architecture has been investigated to enhance UAVs connectivity over the unlicensed spectrum in [31]. The mmWave frequency band is considered for the operation of UAV-based base station in [32] due to the large available bandwidth and reduced antenna size requirement in the band. Multi-beam multi-stream communications using the aerial base station are further studied.

For the ground IoT network, a memory efficient, robust, and reliable communication among ground IoT nodes is required for the effective disaster management. However, there are several loopholes in current IP-based network architecture in terms of caching, mobility, security, and flexibility [18]. We need 
an alternative network architecture that can address these issues. Recently, the unique competitive IP less architecture has been proposed, named as Information-Centric Networking (ICN). ICN architecture consists of retrieval of information (content) based on unique names, regardless of the server location (i.e., IP addresses), channel distribution hierarchy, and application used [33]. There are about eight ICN based architectures proposed in the literature. Some of them are suitable for a static network such as (DONA, NetInf, Mobility First, PURSUIT, CONVERGENCE, and COMET) and the others are for mobile networks, i.e., (Content-Centric Networking (CCN) and NDN) [20,34]. The main aim of all these architectures is to efficiently handle access and distribution of content. These architectures are able to effectively handle link disconnection and interruption during communication through enhanced control over data caching, multi-device communication through replication, and by using different interaction models for sender and receiver. These features of ICN based future internet architecture are well suited for modern systems with dynamic requirements such as UAV-based IoT platform [18].

In [21], the authors proposed a disaster management system based on NDN architecture. In the proposed system, an NDN producer pushes emergency content to nearby consumers. The proposed system achieved low latency and high throughput compared to the legacy NDN architecture. An NDN-based MANET is presented for disaster management in [35] and a proactive routing protocol is proposed for reliable and efficient communication. Experimental results are also provided based on real scenario of smart cars. In [36], an NDN based emergency message delivery mechanism is proposed by using multi-casting. An NDN-based solution is proposed in [37] that uses the satisfied interest table (SIT) along with push-based messages for communication among nodes. The SIT can help nodes to quickly find relative information in a fragmented network that can be useful in scenarios such as disaster management. In [38], the authors proposed an NDN based UAV's communication system in which authors emphasize the importance of light weight ICN (NDN) based communication mechanism in UAVs. Moreover, lightweight interest and data packet payload and the inbuilt security feature of NDN architecture can help the smart gadgets to communicate with each other with increased reliability and less power consumption. These NDN advantages further secure and enhance the IoT-UAV based systems.

In this paper, we propose NDN-based ground IoT mechanism for wildfire management. The trigger based NDN communication in ground IoT network increases the efficiency of the system in terms of reliability and response time of the nodes besides reducing the overall power consumption.

\subsection{Applications}

Applications of UAVs in disaster management vary according to their role during various phases of disaster management and can be broadly classified as shown in Figure 1.

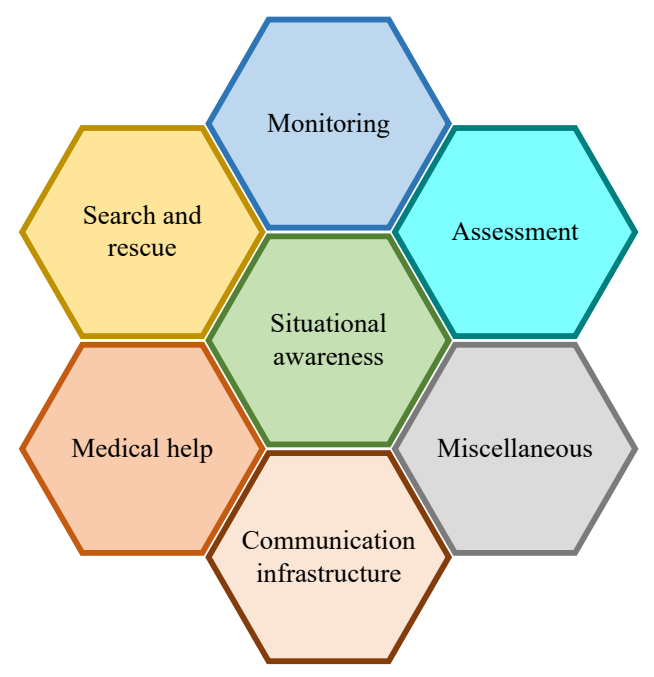

Figure 1. Classification of applications of UAVs-enabled IoT platform in disaster management. 


\subsubsection{Monitoring}

The monitoring of environment through UAVs can help to forecast disaster, prepare for it beforehand, and prevent it. In [9], UAVs are deployed in an area where a disaster can occur to collect aerial imagery and sensor data. An image stitching technology is proposed to detect disaster by analyzing the mapped sensor data on the stitched image. In [8], a mountain fire is monitored in real-time specifically for transmission lines using UAVs. The power consumption of UAVs is optimized by reducing the required number of waypoints to take images of the disaster-hit area and a rapid image processing method is proposed for image stitching. In [11], UAVs are used for real-time data collection from sensors and to forward them to the cloud for further processing and analysis in disaster scenarios.

\subsubsection{Search and Rescue}

UAVs are employed in search and rescue during disaster situations to locate the survivors in the disaster-hit area and provide support. In [12], human wearable IoT devices establish connectivity through UAV mounted base stations in the absence of traditional network infrastructure to send messages for disaster rescue. A multi-UAVs system is considered to search and rescue people affected by natural disaster in [39]. The authors proposed a scheduling scheme to minimize the processing and data transfer time which are critical in search and rescue operations. In [10], a search and rescue system composed of multiple UAVs is studied and a centralized task distribution algorithm is proposed to locate maximum number of survivors in minimum time. Energy efficient mobility model and routing protocol for UAVs in disaster recovery operation are studied in [40] that can adapt to the topology changes while minimizing the network delay. A multiple UAVs autonomous evacuation guidance support system is investigated in [41]. The proposed system decides the evacuation route according to the disaster situation through cooperation between the UAVs.

\subsubsection{Situational Awareness}

Situational awareness implies gathering of information regarding development in a disaster situation, rescue efforts, and the affected people. Situational awareness plays a critical role in decision making involved in disaster management and reduces the response time of rescue operation. Aerial assessment of disaster area using UAVs provides accurate and real-time situational assessment. In [5], a lack of situational awareness in disaster scenarios is studied and it is recommended that the deployment of UAVs along with sensor network in the affected area can provide accurate and timely information required during various phases of disaster management such as planning and disaster studies. Cooperation between the sensor network and UAVs is also considered in [42] to improve the disaster response through timely detection of events and reliable transmission to the data sink. The authors also presented a reliable routing protocol that can dynamically respond to the network topology changes. In [43], a UAV-based remote sensing system is exploited for effective decision support in an emergency situation such as a natural disaster.

\subsubsection{Assessment}

Accurate information about the magnitude and scale of damage inflicted by a disaster is critical for efficient disaster management. UAVs collect videos, images, and sensors data from the affected area which is further processed and analyzed for damage assessment. In [44], UAVs are used to estimate the damage to a power network due to some extreme weather event. The authors provided a mathematical framework for efficient damage assessment and also studied the optimal location and trajectory of the UAV for assessing damages. UAVs are used to collect high-resolution videos and images from inaccessible sites after an earthquake to estimate damage in [45]. The authors developed three dimensional (3D) models based on these collected data using the structure-from-motion technique to evaluate damage situation. In [46], UAV images of a site before and after destruction are used to estimate the amount of damage. UAV-based mapping is utilized for quick assessment of the damage 
caused by volcano eruption through change detection analysis in [47]. In [5], integration of WSN with UAVs is considered for disaster assessment where UAVs gather data from the affected area and relay it to the data center in real-time.

\subsubsection{Medical Help}

UAVs can be used for emergency medical response in the disaster-hit area to provide medical help and supplies. Since time is critical in the case of medical emergency and chances of survival rapidly decrease with time, UAVs can be used to quickly reach people in need before the other medical help arrives. In [48], UAVs are used to provide medical aid with the help of onboard sensors and the robotic arm. Particularly, the authors studied the case of cardiac arrest and present a framework for handling such a medical emergency. The health of soldiers in the battlefield is monitored using UAVs and wearable sensors in [49]. The objective is to efficiently direct medical help in order to save soldiers lives. In [5], UAVs are considered for delivering essential medical supplies in disaster-hit areas with damaged or destroyed transport infrastructure.

\subsubsection{Communication Infrastructure}

In a disaster scenario, the conventional communication infrastructure may get destroyed. In this case, UAVs can be incorporated to fill the coverage holes. Drone-based small cell deployment is investigated to fill the coverage holes in a cellular network post-disaster in [13]. The authors also proposed a clustered deployment of drone-based small cells in the disaster-hit area. In [14], the location of the UAV-mounted BS is optimized to improve throughput and coverage performance. The energy efficient path for the UAV to serve a maximum number of users in post-disaster communication under limited battery capacity is investigated in [16]. The authors of [15] proposed a distributed NOMA scheme for emergency communication using UAVs. It jointly optimizes the channel assignment, hovering time, and power budget of UAVs to maximize the access ratio and sum rate of IoT devices in disaster-affected areas. UAV-mounted base stations are adopted in [12] to facilitate M2M communication between human wearable IoT devices for disaster rescue. A resource allocation scheme is proposed to improve user scheduling and energy efficiency of UAV mounted BSs. The flow of critical information using UAVs in the absence of a legacy communication network in the event of a disaster is investigated in [50]. The authors employed multiple UAVs with specialized payloads to scan the affected area for survivors and take images for decision support scenarios.

\section{Architecture and Key Challenges}

In this section, we present the architecture of UAV-enabled IoT platform for disaster management. We also present key challenges for the successful deployment of IoT platform for disaster management.

\subsection{Architecture}

Figure 2 presents the architecture of UAV-enabled IoT platform for disaster management. The IoT platform consists of three main components: (i) ground IoT network; (ii) ground and aerial connectivity of ground IoT network and UAVs; and (iii) data analytics. The information regarding disaster and emergency situation can be collected from the ground IoT network and patrolling UAVs. Moreover, UAVs can act as base station or relays in disaster situation when the wireless infrastructure is damaged. Current trends in research and implementation are to use human controlled UAVs for IoT platforms. Moreover, these UAVs are not connected via cellular network for data collection and relay. It is important to incorporate autonomous UAVs in IoT platform for disaster management as it offers many advantages such as improved tactical decision making and response time. However, these advantages come with challenges including privacy issues, crashes, close encounters with manned vehicles (aircrafts), shoot down, and inappropriate use [51]. For example, according to Transport Canada, the number of close encounters with aircraft has tripled since 2014 [52]. In a recent incident in 
Edmonton, an aircraft pilot reported that he noticed a drone 2000 feet below his left wing in 2018. It is extremely dangerous when UAVs are flying that close to an aircraft.

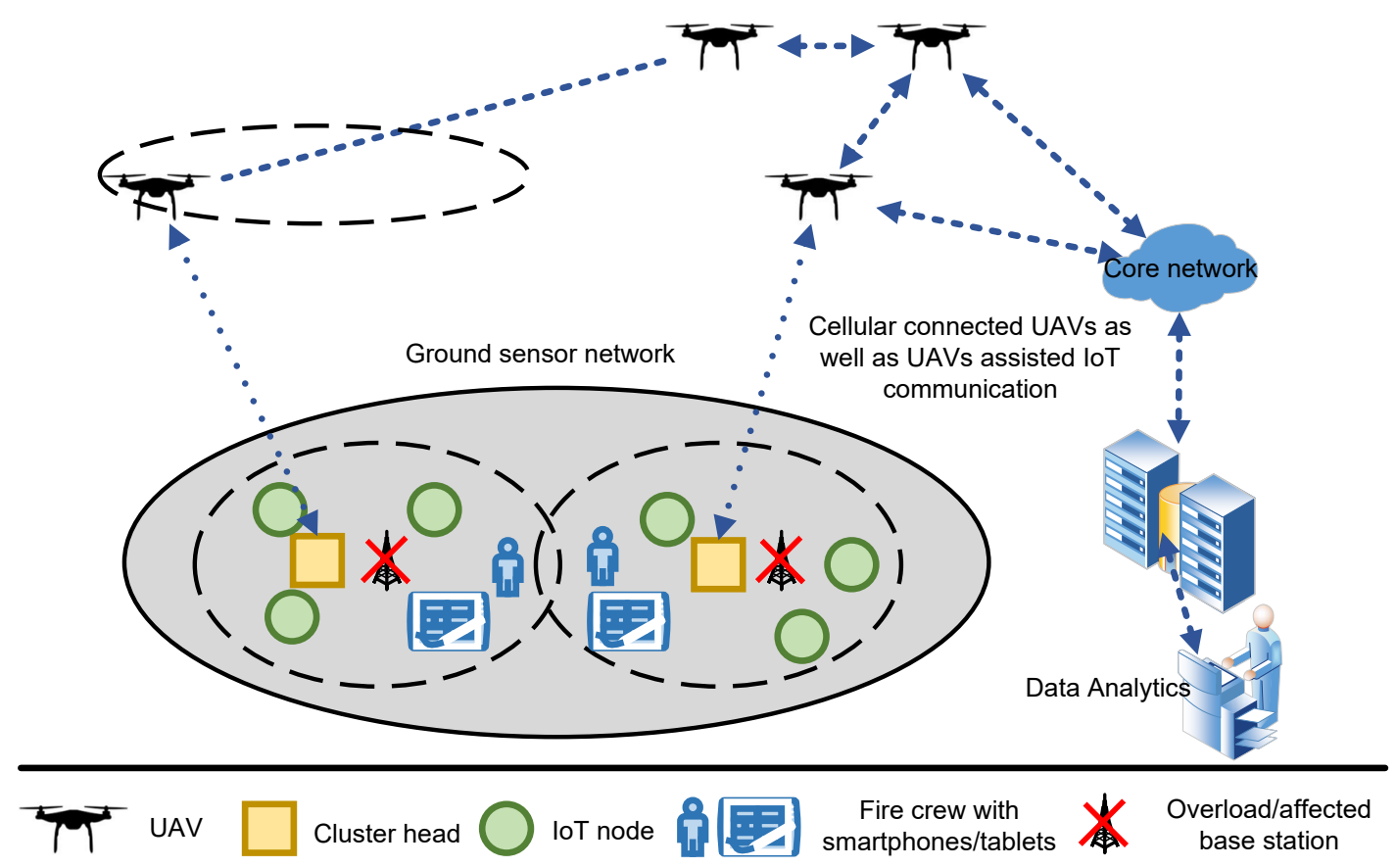

Figure 2. Overview of UAV-enabled IoT platform for disaster management.

The loss of connectivity is reported as the major cause behind the UAV crashes [52]. Therefore, wireless connectivity is the key to safe operation of UAVs. Earlier generations of cellular networks were solely designed to support users on the ground. Beyond 5G cellular networks need to be investigated to have inherent features to support both aerial and ground users. In addition, how UAVs can effectively act as a base station in the affected region should also be studied. Particularly, focus should be given on spectrum allocation and interference management to improve the performance of the IoT platform and to avoid any unexpected interruptions in communication. Further, a framework should be defined based on the input from wildfire management department and the rules and regulations of Transport Canada (or similar authorities in other countries) for the operations of UAVs.

The data collected by the UAVs need to be analyzed using machine learning techniques to effectively predict the wildfire ahead of time. This, for example, can help to control the wildfire by determining the rate and direction of fire. In addition, the ground sensors data can be used to predict the forest fire in advance. Therefore, data collection using UAVs (from the ground IoT network and from the patrolling $\mathrm{UAVs}$ ) and their analysis is a unique research area especially for wildfire management. Further, data analytics can provide great insight to reduce the socioeconomic damage caused by wildfire. Data analysis can certainly help to predict the fire which can significantly reduce the ongoing total cost. Machine learning and artificial intelligence techniques can also play a significant role in design optimization to enhance the performance of UAV-enabled IoT platform. They can be used to validate the disaster management architecture under realistic wildfire scenarios.

For data acquisition from ground IoT network, routing path and deployment of UAVs has been investigated in [17]. Flight performance of UAV is also investigated in [53]. The authors studied data mapping and efficiency of multi-UAV system in terms of the number of UAVs required to serve a disaster area. The role of data analytics is highlighted for wildfire prevention to reduce environmental as well as socioeconomic losses in [54]. Machine learning and artificial intelligence can play a crucial role to predict wildfire. A wildfire prediction model is proposed in [55] to determine the direction and rate of fire spread. The advent of UAVs has shifted the focus of aviation laws and regulations enforcement [56]. However, it is challenging to enforce laws for the autonomous UAVs where there 
is no pilot. Researchers are investigating law enforcement for autonomous UAVs and Transport Canada has also defined Canadian aviation regulations. It is important to design and deploy IoT platform while considering the requirements of wildfire management department and satisfying the rules of Transport Canada (or similar authorities in other countries). Despite this available literature, wireless connectivity, data analytics, and law enforcement still need to be comprehensively included in the UAVs enabled IoT platform for wildfire management to enable effective deployment and easy adaptation by the wildfire management department.

The architecture of UAV-enabled IoT platform can help in multiple ways for wildfire management: (i) fire crew and people in the affected areas will have coverage through UAVs enabling their long-range communication; (ii) autonomous UAVs can spot forest fires at early stages before they get out of control and also identify people in need in the affected areas; (iii) the ground IoT network can be helpful to collect useful information in wildfire areaa; and (iv) many data collected from the ground and UAV system can be relayed to the cloud infrastructure where the data can be analyzed to improve the performance of the system and predict future conditions, in addition to providing recommendation to the wildfire management authorities.

\subsection{Challenges}

We categorize challenges associated with the UAV-enabled IoT platform for disaster management into four areas: (i) efficient connectivity solutions; (ii) data analytics; (iii) law enforcement and technology; and (iv) sensor network deployment.

The flight time of a UAV is limited by its battery capacity that needs to be charged at regular intervals. It raises various challenges for the emergency communication systems where the UAVs are utilized in disaster recovery network. The key issues include seamless handover between the UAVs to maintain user sessions, managing charging cycles of UAVs, and maintaining uninterrupted connectivity with the radio access network. Selecting the optimal position for a UAV-based data relay is also a major challenge that is complicated by the lack of knowledge about the user distribution and damaged network infrastructure in the disaster area. Moreover, due to the movement of UAVs, interference management in such networks is another main concern. As time is critical in rescue operations and since the topology of UAV based emergency and public safety network is not fixed, efficient dynamic routing protocols are required that can rapidly adapt to the topology changes to maintain connectivity and minimize the network delay. There are also unique challenges in terms of service requirement of different users in a disaster scenario. The traffic post-disaster is mostly bursty in nature and most users are concentrated in a small area, which can overload the network. For the first responders and rescue personnel, the network must be able to support broadband data rates such that the critical voice, video, data and control information can be sent and received.

UAV-enabled IoT platforms for disaster management typically generate large-scale streaming data from heterogeneous sources tagged with time and space information. Ubiquitous knowledge of disaster further requires data fusion and sharing, which is especially important in time-sensitive IoT applications such as wildfire management. Traditional IoT data analytics and learning methods are not suitable to handle such a task. This calls for fast and streaming data analytics to predict future insights and to enable timely decisions that are critical in case of a disaster scenario. UAV-enabled disaster management systems in the literature typically consider cloud infrastructure for data analysis where the UAVs relay sensory data to the cloud, which communicates back the decision. This adds extra latency to the response time which can have a serious effect in certain cases. To minimize delay, fast data analytics close to the source is required.

Legal and technological challenges associated with the deployment of UAVs are mainly concerned with their impact on the privacy of the victims and administrative issues such as the laws governing the use of UAVs. These government regulations vary with country and define size, range and maximum flight height of the UAVs along with their use of airspace to ensure safe operation in the environment. Data protection is a main concern with the use of UAVs as the data acquired from various sensors could 
possibly include sensitive information. Therefore, UAV-based disaster management system must have a high level of integrity and secure data links to avoid misuse. Security is another major issue with UAV-based systems as they are vulnerable to hacking and hijacking. This requires implementation of high standards of encryption and authentication.

The deployment of the sensor network is still a major concern as it affects coverage and connectivity. Sensor node placement is particularly challenging in disaster management because accurate environmental information is required for useful results. The problem is further complicated as the sensor nodes are subject to random failures due to factors such as harsh environment, hardware failure, and battery depletion. For reliable sensor network, nodes should be deployed to get maximum coverage with minimum overlap and excellent connectivity with the sink. It is also essential to have redundant nodes in the network to improve the reliability of the sensor network. However, the total deployment cost also needs to be minimized. Most of the literature on sensor network deployment deals with two dimensional (2D) environments, whereas the deployment environment in the case of UAV-enabled IoT platform is $3 \mathrm{D}$, which is even more complex.

\section{Case Study: NDN Architecture for Ground IoT Network}

The information regarding disaster situation can be collected using the ground IoT network. Compared to conventional fire detection and alarm systems on the ground, sensors network for IoT can be more effective, efficient and reliable for disaster management scenarios. The NDN architecture is a potential candidate to achieve these goals. Here, our main objective is to handle the ground IoT network for wildfire scenario using NDN architecture. NDN is evolving as the most likely or prominent information-centric future Internet architecture [18,21,57]. NDN is a content retrieval architecture rather than a host-centric network in both wired and wireless domains. Moreover, its unique and innovative functionalities such as caching ability inside the node, name-based communication and routing schemes favor the basic requirements of IoT architecture. An IoT-based communication mechanism is quite different from today's Internet due to the existence of resource-dependent devices, immense volume of small data exchange and diversity in traffic types $[18,58]$. Furthermore, it also has loopholes in the traditional end-to-end communication mechanism. In other words, it emphasizes on "what" are the required data instead of "where" are the required data $[18,19]$. NDN describes a robust, flexible and straightforward output-driven communication mechanism based on the interchange of two unique packet types, namely "Interest" and "Data" packets, which carry categorical, application-explicit data content names. NDN ensures content integrity and validity by sponging the data publisher's signature and other relevant verification information constraints in each data packet. Moreover, based on local requirements, a complete or partial subset of the overall network nodes can cache contents to increase the data retrieval rate while decreasing the overall network overhead [34,59]. Each NDN node keeps and preserves three data structures $[18,20,58,59]$ :

- Content store (CS): CS maintains short-term caching of incoming data packets.

- Forwarding information base (FIB): FIB maintains the path between interests and actual data.

- $\quad$ Pending interest table (PIT): PIT keeps track of the unsolicited interest(s) that are not yet satisfied with a corresponding response data packet.

Moreover, each NDN consumer node publishes an interest to find appropriate data through producer node in the network $[34,58]$. In the first step, it explores for a longest name prefix match in its CS. The node reply-back "Data" packet to the corresponding arriving interface of processed interest only if an exact match is found. Otherwise, "Interest" packet is rejected or discarded if there is a matching PIT entry found in the data structure. This phenomenon is executed when an equivalent interest request has already dispatched. In contrast, if it is not the situation, a new PIT entry is established in its PIT, and finally, the "Interest" is further accelerated to the path stored in the FIB structure. In addition to this, "Data" packets track the series of PIT entries back to the consumers. 
Hence, if a match does not exist in the PIT structure, then the "Data" packet is categorized as unsolicited and is dropped $[59,60]$.

Wildfire situations produce multiple network fragmentations. Therefore, to retrieve the data from such a fragmented network, NDN mechanism is very effective due to its data-caching facility inside each node structure. Moreover, it also ensures minimal delay between consumer and producer NDN nodes, especially in a disaster situation. To validate this point, it does not need to republish the interest message in the case of network fragmentation. It can retrieve data from the nodes data structure hierarchy included in the network path [21,37].

\subsection{Name-Based Push/Pull Mechanism}

Although the existing NDN architecture, particularly for mobile networks, provides inefficient consumer-centric support for repossessing data, to retrieve data from a respective producer, the NDN consumer node must broadcast an "Interest" packet in the whole network. As the consumer must wait to get the "Interest" satisfied from a producer data response, it causes a long delay between nodes communication. In contrast, in a wildfire situation, nodes are scattered due to the wildfire. Therefore, we need a robust and efficient communication mechanism to incorporate a large volume of data within no time [21]. However, the traditional NDN pull-based communication mechanism fails to provide such a rapid response [61]. Therefore, a unique competitive push-based critical data forwarding mechanism is proposed to handle emergency situations [62] that allows nodes with critical content to broadcast a unique beacon message to their one-hop neighbor's distance. In reply to that beacon message, the neighbors make short-term entries in their PIT data structure, and hence, in this way, the upcoming critical content chunks are cached instead of being discarded due to unsolicited chunks treatment. The existing NDN architecture is modified to manage PIT content systematically and to enable pushing of content where essential [21,61]. The application permits the integration of the preeminent forwarding and routing strategies to suit a disaster situation. However, the traditional IP infrastructure cannot deal with disastrous situations, especially in the case of network partition or fragmentation. This is mainly because of the Transmission Control Protocol (TCP/IP) protocol on end-to-end communication [21,37,61,62].

\subsection{Proposed Methodology}

Figure 3 shows the method adopted to achieve the NDN-based wildfire objective. Here, we use multiple sensors such as flame and smoke sensors to detect a wildfire disaster. However, these two sensors are attached with the controller with WiFi module (IEEE $801.11 \mathrm{~b} / \mathrm{g} / \mathrm{n}$ ) to transmit the critical information towards all nearby consumer nodes. We use different consumer nodes (such as Consumers A, B, C, ..., H) to gather "Interest" messages from respective consumers that ultimately reach the desired destination with the help of a centralized server. Each consumer node collects information in its network domain, which finally interface with the central server. Moreover, the nodes continuously send their collected data (values of sensors) to the server. The significant feature of the application and its design is the merging of hybrid push-pull-based communication to enable reliable and efficient message propagation in the network. In our wildfire scenario, we need a push-based communication mechanism to increase the robustness and reliability of a disaster management system. On the other hand, the pull-based communication system is enabled when we have a disaster-free situation. Hence, the recipient has control over what it receives.

From the security aspect, it also has a large time window to verify the identity of the sender, from the time elapsed between the demand request and receipt of content at the receiving end. According to the push-pull-based communication mechanism, whenever a client sends an interest packet to get the server for checking the status of nodes, it replies with an acknowledgment signal. It then checks the values of the respective node, i.e., whether they are within the threshold limit or not. Furthermore, a particular calibrated value for every sensor is set already. If the values coming from the sensor of the respective node are within the threshold, it sends the values to the respective consumer. 
On the other hand, if the value exceeds the threshold, the server generates an alert by broadcasting a message via an NDN Android application (App), indicating that damage could occur due to fire. Figure 4 shows the block diagram of the NDN wildfire detection mechanism.
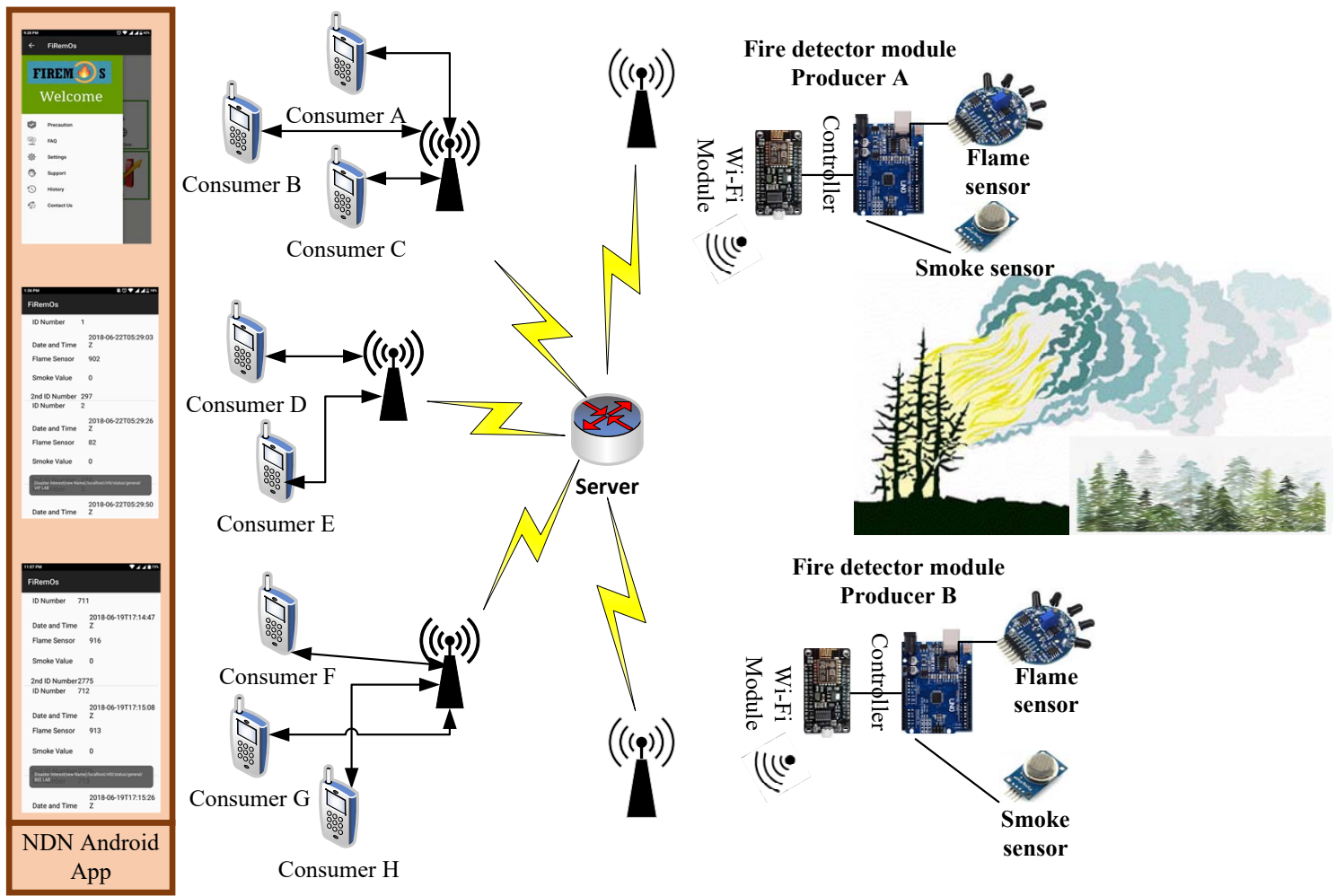

Figure 3. The NDN IoT-based wildfire scenario.

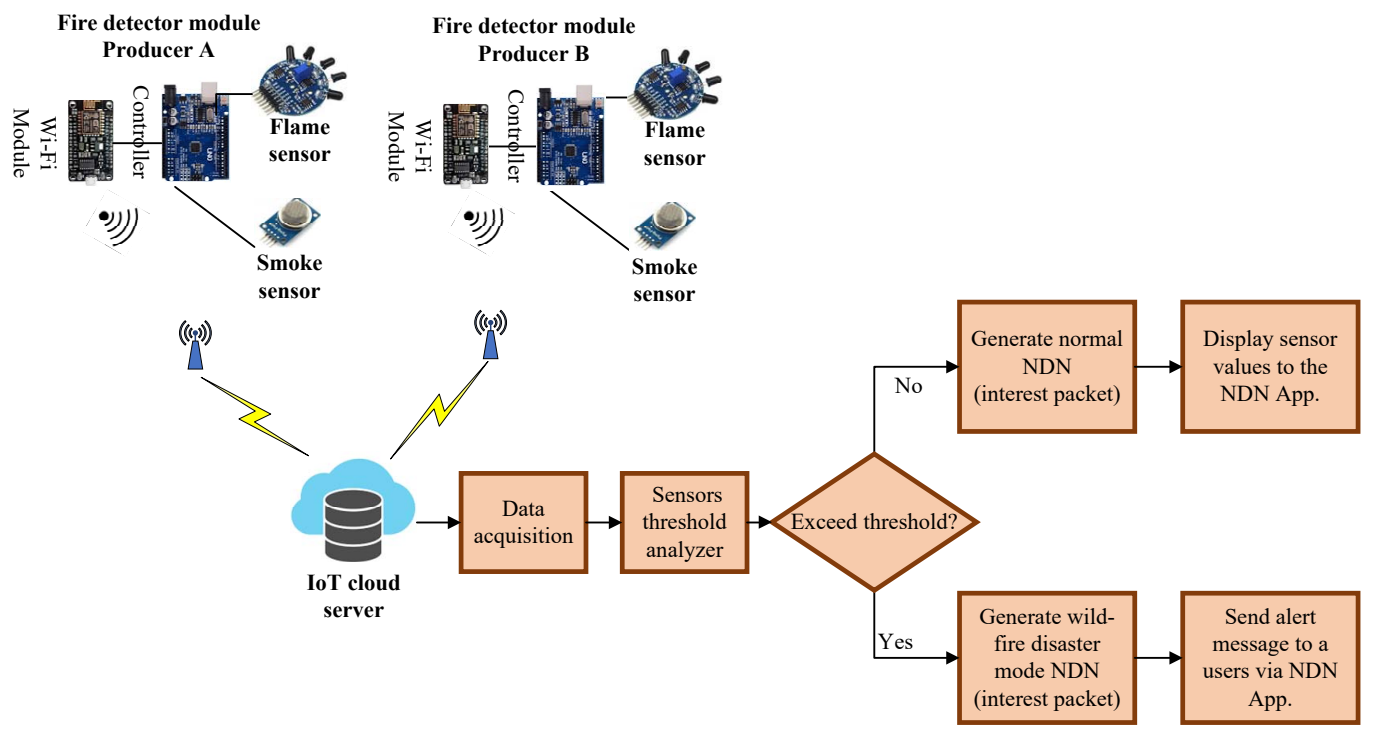

Figure 4. Block diagram of teh NDN wildfire detection mechanism.

In a wildfire scenario, NDN architecture becomes more reliable as compared to IP-based system due to the presence of a three-level data caching structure (CS, PIT and FIB). A disastrous state most likely disconnects the network into multiple fragments, thus communication is the most challenging task, which NDN provides with robustness and with esteem surety. Furthermore, in NDN mechanism, we do not need to retransmit data in the case of network fragmentation directly from the source; instead, 
we use on the path nodes to act as source to transmit information stored in their data structures. Further, we consider with two parameters in wildfire scenario, i.e., average delay (transmission + propagation) between nodes and the reliability of the system. We use an NFD library in our controller and an Android App, which provide us the functionality of the NDN mechanism and generate log files. We tested our modules according to the different disaster impacts, i.e., $10 \%, 20 \%, 30 \%, 40 \%$ and $50 \%$, with different numbers of nodes.

The hardware implementation consisted of flame and smoke sensors that were connected to the micro-controller that sent the data to the IoT cloud server using the WiFi module, as shown in Figure 5. The Arduino Mega was used in fire detection module, which is a micro-controller board based on the ATmega2560. The MQ-2 smoke sensor and flame/fire sensor (YG1006) were used for fire detection. We used cellular and WiFi technologies for the fire detection module. The values of the sensors were continuously monitored and, when they exceeded the threshold value, an alert message was broadcasted to all users through the NDN-based Android App. The pull-push-based mechanism was followed for broadcasting the message.

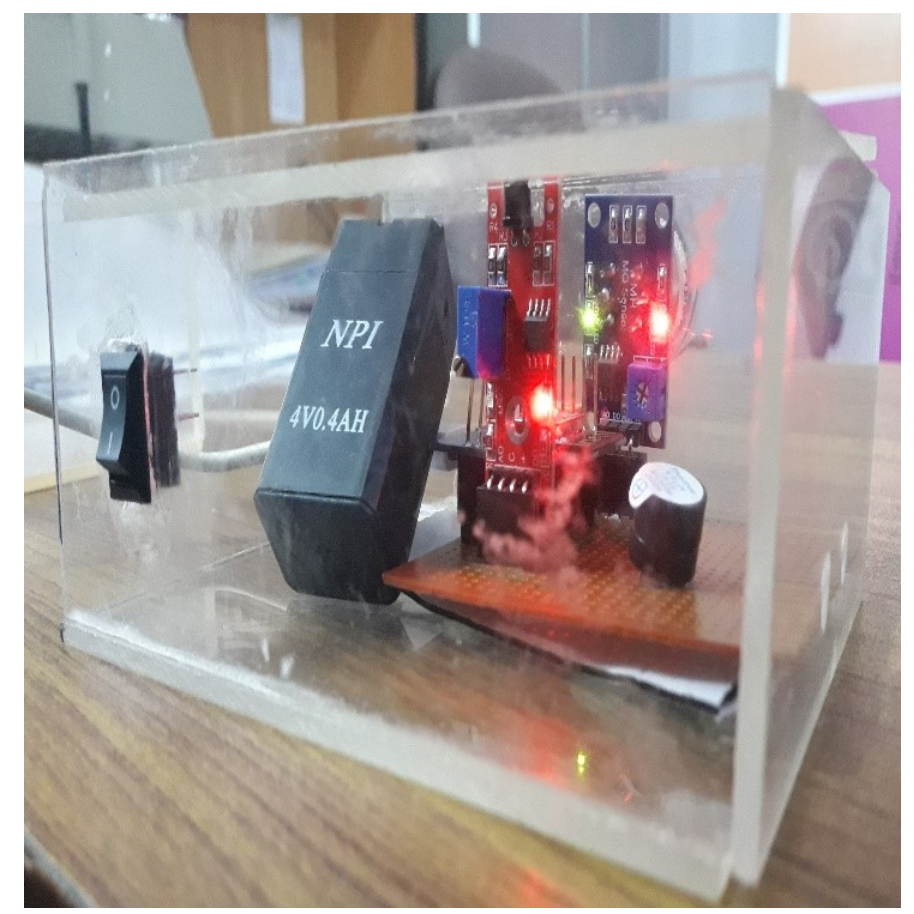

Figure 5. Implementation of the fire detection module.

\subsubsection{Results and Discussion}

The evaluation of the pull-push NDN-based real-time wildfire disaster detection mechanism was carried out through nodes average response time (ART) and communication architecture reliability (CAR).

\subsubsection{Average Response Time (ART)}

Figure 6 shows the relationship between the total number of nodes with respect to ART in wildfire disaster scenario. It compares two architectures, i.e., IP architecture (IP Arch.) and real-time proposed NDN (Pull-Push) architecture (NDN Arch.). The total number of NDN nodes were varied from 10 to 80 . The ART is the ratio of the sum of total delay, i.e., propagation and transmission delays to the total time taken. It can be calculated as:

$$
A R T=\frac{\sum^{N}\left(\tau_{p}+\tau_{t}\right)}{T}
$$


where $\tau_{p}$ and $\tau_{t}$ are the propagation and transmission delays, respectively; $T$ is the total time taken in transmission; and $N$ is the total number of consumer nodes. The result depicts that the proposed real-time NDN (Pull-Push) mechanism outperforms IP architecture in a wildfire disaster situation. Furthermore, the reason behind this overwhelming performance of NDN architecture is the unique data caching ability inside nodes, which is one of the major drawbacks of the traditional IP architecture. In wildfire, network nodes are scattered due to disaster; therefore, in the case of TCP/IP architecture, the source retransmits the information that is lost. On the other hand, NDN architecture recovers the required information from the NDN node's data structure, i.e., CS, PIT, and FIB. In this way, the NDN-based message communication in wildfire performs exceptionally as tit does not need to retransmit the information. Moreover, the IP architecture has higher network delay than NDN due to the retransmission of information from the source.

\section{IP Arch. $\quad$ NDN Arch.}

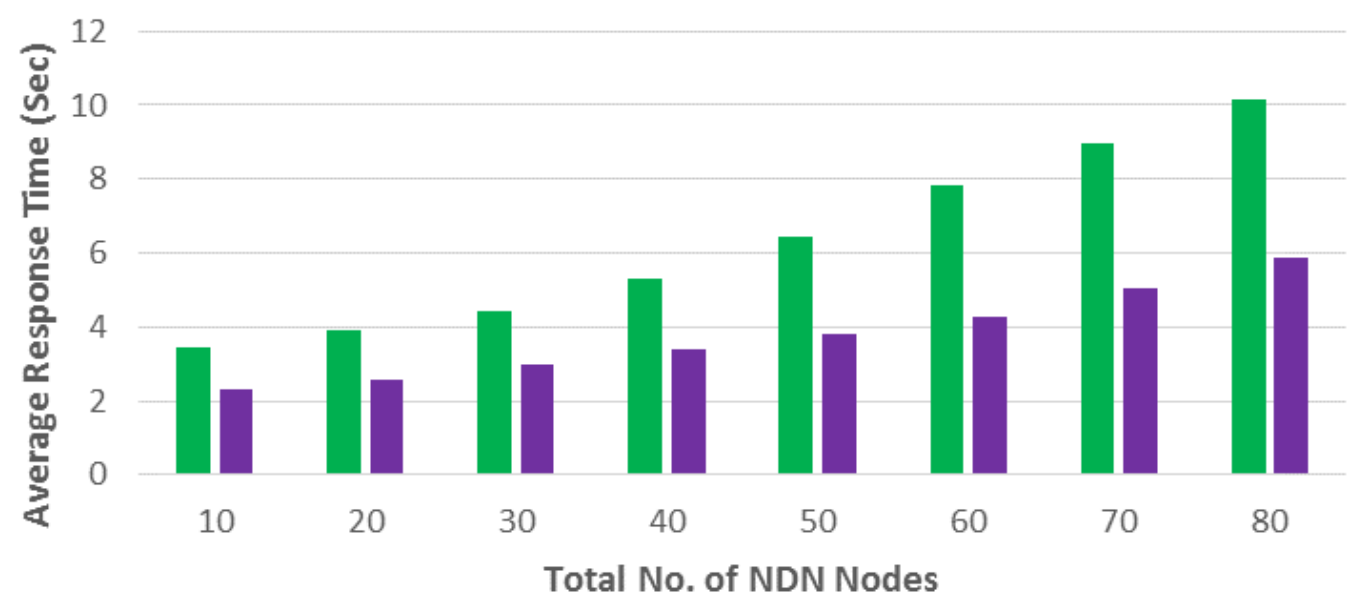

Figure 6. Nodes average response time in wildfire scenario.

Table 1 depicts the communication reliability of both architectures, i.e., (IP architecture and NDN architecture). We evaluated both architectures based on the percent wildfire disaster impact for 100 nodes, e.g.,

- $10 \%$ disaster impact means 10 out of 100 nodes are defected; and

- $20 \%$ disaster impact means 20 out of 100 nodes are defected.

Table 1. Communication architecture reliability.

\begin{tabular}{ccc}
\hline \% Disaster Impact & IP Architecture & NDN Architecture \\
\hline 10 & $\checkmark$ & $\checkmark$ \\
20 & $\checkmark$ & $\checkmark$ \\
30 & $\checkmark$ & $\checkmark$ \\
40 & $\boldsymbol{x}$ & $\checkmark$ \\
50 & $\boldsymbol{x}$ & $\checkmark$ \\
\hline
\end{tabular}

However, as the disaster impact increases beyond 30\%, the traditional IP architecture is unable to communicate between the nodes due to massive network damage. In contrast, NDN architecture still retains its message communication flow even at 50\% disaster impact due to the caching ability inside the nodes. By adopting IP based mechanism, we do not have any suitable technique to recover information in a disastrous situation and the information is lost when the disaster impact crosses $50 \%$. On the other hand, in NDN, we do not rely on end to end communication. Information is stored inside each node (CS, PIT and FIB) that is along the path and, therefore, we have multiple nodes to 
recover information from instead of only the originator node as in case of the IP based system. Hence, with NDN, information can be recovered from any node along the path, even if the disaster impact crosses $50 \%$. By considering the above results and analysis, it is concluded that the NDN architecture outperforms the IP architecture in real time wildfire situation and the NDN mechanism is more reliable, effective, and robust as compared to the traditional communication approaches.

\section{Conclusions and Future Research Directions}

Disaster situations require a flexible and reliable information dissemination mechanism to facilitate communication. A UAV-enabled IoT platform can significantly enhance the performance and reduce the cost for disaster management scenarios. In this paper, we present an architecture for a UAV-enabled IoT platform for disaster management and highlighted the key challenges in the area including wireless connectivity, data analytics, ground IoT network deployment, and experimental validation. We then present a case study for the ground IoT network in which NDN architecture was used resulting in a reduced delay for decision making, increased efficiency of content delivery, and greater data security. The improved performance of the ground IoT network is demonstrated through the results of the case study (NDN architecture for IoT platform).

In this paper, we only address the ground IoT network. Future work is to consider communication technologies for ground and aerial connectivity, and data analytics. There are several open research areas in this area that need attention. Here, we briefly discuss four main areas.

\subsection{Efficient Wireless Connectivity Solutions}

The aim is to provide ubiquitous connectivity to both aerial users and the ground IoT network. Energy, spectrum, and computation resource should be optimized, which have a high impact on the performance of the IoT platform in dynamic conditions. The focus should be given to spectrum allocation using CR technology, which is shared between aerial and ground users. The optimization solution for patrolling UAVs as well as UAVs acting as base stations to provide connectivity in affected areas should also be studied while satisfying QoS requirements of heterogeneous users. Interference mitigation techniques should be investigated including multi-cell cooperation and NOMA. Further, artificial intelligence, machine learning, and deep learning techniques could be considered for design optimization approaches to enhance the performance of a UAV-enabled IoT platform. These can include linear/polynomial models, support vector machines, and complex neural networks.

\subsection{Data Analytics}

The sensor network for IoT on the ground and patrolling UAVs will collect the data and forward it to the data management center using the cellular network. The data from multiple sensor nodes on the ground should be aggregated and properly structured using extract, transform, and load (ETL) approaches. Novel data predication models and visualization techniques should be studied to analyze and forecast the rate and direction of fire spread. A real-time data recommendation system should be proposed and the results can be made available for the wildfire management team and general public through our developed mobile application.

\subsection{Law Enforcement and Technology}

Legal enforcement issues are arising with the development of technology, particularly autonomous UAVs. It is extremely important to study the rules and regulations of the regulatory authority of the country (e.g., Transport Canada) for the operations of UAVs. There is an increased concern of safety and privacy for UAVs operation. Therefore, it would be a great contribution to define a framework based on rules and regulations of Transport Canada (or a similar authority in another country) for using UAVs. The input from different stakeholders including fire management department and regulatory authority should be integrated to design realistic scenarios for fire management as well as UAVs patrolling and data collection scenarios. 


\subsection{Sensor Network Deployment and Experimental Validation}

The survey of the area is of paramount importance for UAVs operation and deployment of ground IoT network for IoT to collect information (such as temperature, humidity, etc.) from forests where we can expect a fire. The ground IoT network will be connected to UAVs wirelessly and UAVs will transfer the collected information to the cloud infrastructure. In addition, experimental validation is key in real wildfire management scenarios. The development/availability of this experimental setup will certainly allow us to investigate more complex scenarios in the future.

Author Contributions: W.E conceived the presented idea and provided the architecture provided in this paper. M.A.A., W.E., and F.I. supervised the finding/implementation of this research work. W.E. wrote the paper with close participation of S.S. and A.H. All the authors took part in revising the article and provided critical feedback in shaping the research and discussed results.

Funding: This research was funded in part by the Cluster Research Project (R16083) and RIF (R18055), Zayed University, Dubai, United Arab Emirates. This research was also supported by Thompson Rivers University, Kamloops, Canada.

Conflicts of Interest: The authors declare no conflict of interest.

\section{References}

1. Wildfire Response. Available online: https://www2.gov.bc.ca/gov/content/safety/wildfire-status/aboutbcws/wildfire-response (accessed on 21 May 2019).

2. Ejaz, W.; Ibnkahla, M. Machine-to-machine communications in cognitive cellular systems. In Proceedings of the 2015 IEEE International Conference on Ubiquitous Wireless Broadband (ICUWB), Montreal, QC, Canada, 4-7 October 2015; pp. 1-5.

3. Ejaz, W.; Ibnkahla, M. Multiband spectrum sensing and resource allocation for IoT in cognitive $5 \mathrm{G}$ networks. IEEE Internet Things J. 2018, 5, 150-163. [CrossRef]

4. Ray, P.P.; Mukherjee, M.; Shu, L. Internet of things for disaster management: State-of-the-art and prospects. IEEE Access 2017, 5, 18818-18835. [CrossRef]

5. Erdelj, M.; Natalizio, E.; Chowdhury, K.R.; Akyildiz, I.F. Help from the sky: Leveraging UAVs for disaster management. IEEE Pervasive Comput. 2017, 16, 24-32. [CrossRef]

6. Mozaffari, M.; Saad, W.; Bennis, M.; Debbah, M. Mobile unmanned aerial vehicles (UAVs) for energy-efficient internet of things communications. IEEE Trans. Wirel. Commun. 2017, 16, 7574-7589. [CrossRef]

7. Wildfire Averages. Available online: https://www2.gov.bc.ca/gov/content/safety/wildfire-status/aboutbcws/wildfire-statistics/wildfire-averages (accessed on 21 May 2019).

8. Zhang, W.; Yu, H.; Yan, Z.; Xu, J. Study on disaster monitoring technology of mountain fire based on UAV transmission line inspection. In Proceedings of the IEEE International Conference on Unmanned Systems (ICUS), Beijing, China, 27-29 October 2017; pp. 400-403.

9. Yim, J.; Park, H.; Kwon, E.; Kim, S.; Lee, Y.T. Low-power image stitching management for reducing power consumption of UAVs for disaster management system. In Proceedings of the 2018 IEEE International Conference on Consumer Electronics (ICCE), Las Vegas, NV, USA, 12-14 January 2018; pp. 1-3.

10. Alotaibi, E.T.; Alqefari, S.S.; Koubaa, A. LSAR: Multi-UAV Collaboration for Search and Rescue Missions. IEEE Access 2019, 7, 55817-55832. [CrossRef]

11. Choksi, M.; Zaveri, M.A.; Kumar, J.S.; Pandey, S.K. Cloud-based real time data acquisition in IoT environment for post disaster management. In Proceedings of the 9th International Conference on Computing, Communication and Networking Technologies (ICCCNT), Bangalore, India, 10-12 July 2018; pp. 1-6.

12. Liu, X.; Ansari, N. Resource allocation in UAV-assisted M2M communications for disaster rescue. IEEE Wirel. Commun. Lett. 2018, 8, 580-583. [CrossRef]

13. Hayajneh, A.M.; Zaidi, S.A.R.; McLernon, D.C.; Di Renzo, M.; Ghogho, M. Performance analysis of UAV enabled disaster recovery networks: A stochastic geometric framework based on cluster processes. IEEE Access 2018, 6, 26215-26230. [CrossRef]

14. Merwaday, A.; Tuncer, A.; Kumbhar, A.; Guvenc, I. Improved throughput coverage in natural disasters: Unmanned aerial base stations for public-safety communications. IEEE Veh. Technol. Mag. 2016, 11, 53-60. [CrossRef] 
15. Liu, M.; Yang, J.; Gui, G. DSF-NOMA: UAV-Assisted Emergency Communication Technology in a Heterogeneous Internet of Things. IEEE Internet Things J. 2019, 6, 5508-5519. [CrossRef]

16. Lin, Y.; Wang, T.; Wang, S. UAV-assisted emergency communications: An extended multi-armed bandit perspective. IEEE Commun. Lett. 2019, 23, 938-941. [CrossRef]

17. Mozaffari, M.; Saad, W.; Bennis, M.; Debbah, M. Mobile Internet of Things: Can UAVs provide an energy-efficient mobile architecture? In Proceedings of the IEEE global communications conference (GLOBECOM), Washington, DC, USA, 4-8 December 2016; pp. 1-6.

18. Baccelli, E.; Mehlis, C.; Hahm, O.; Schmidt, T.C.; Wählisch, M. Information centric networking in the IoT: Experiments with NDN in the wild. In Proceedings of the 1st ACM Conference on Information-Centric Networking, Paris, France, 24-26 September 2014; pp. 77-86.

19. Arshad, S.; Azam, M.A.; Ahmed, S.H.; Loo, J. Towards information-centric networking (ICN) naming for Internet of Things (IoT): The case of smart campus. In Proceedings of the International Conference on Future Networks and Distributed Systems, Cambridge, UK, 19-20 July 2017; p. 41.

20. Akbar, M.S.; Khaliq, K.A.; Rais, R.N.B.; Qayyum, A. Information-centric networks: Categorizations, challenges, and classifications. In Proceedings of the 23rd Wireless and Optical Communication Conference (WOCC), Newark, NJ, USA, 9-10 May 2014; pp. 1-5.

21. Hannan, A.; Arshad, S.; Azam, M.; Loo, J.; Ahmed, S.; Majeed, M.; Shah, S. Disaster management system aided by named data network of things: architecture, design, and analysis. Sensors 2018, 18, 2431. [CrossRef] [PubMed]

22. Huo, Y.; Dong, X.; Lu, T.; Xu, W.; Yuen, M. Distributed and multi-layer UAV networks for next-generation wireless communication and power transfer: A feasibility study. IEEE Internet Things J. 2019. [CrossRef]

23. Lin, X.; Yajnanarayana, V.; Muruganathan, S.D.; Gao, S.; Asplund, H.; Maattanen, H.L.; Bergstrom, M.; Euler, S.; Wang, Y.P.E. The sky is not the limit: LTE for unmanned aerial vehicles. IEEE Commun. Mag. 2018, 56, 204-210. [CrossRef]

24. Azari, M.M.; Rosas, F.; Chiumento, A.; Pollin, S. Coexistence of terrestrial and aerial users in cellular networks. In Proceedings of the IEEE Globecom Workshops (GC Wkshps), Singapore, 4-8 December 2017; pp. 1-6.

25. Azari, M.M.; Rosas, F.; Pollin, S. Reshaping cellular networks for the sky: Major factors and feasibility. In Proceedings of the 2018 IEEE International Conference on Communications (ICC), Kansas City, MO, USA, 20-24 May 2018; pp. 1-7.

26. Lai, C.C.; Chen, C.T.; Wang, L.C. On-Demand Density-Aware UAV Base Station 3D Placement for Arbitrarily Distributed Users with Guaranteed Data Rates. IEEE Wirel. Commun. Lett. 2019, 8, 913-916. [CrossRef]

27. Basharat, M.; Ejaz, W.; Naeem, M.; Khattak, A.M.; Anpalagan, A. A survey and taxonomy on nonorthogonal multiple-access schemes for 5 G networks. Trans. Emerg. Telecommun. Technol. 2018, 29, e3202. [CrossRef]

28. El-Mougy, A.; Ibnkahla, M.; Hattab, G.; Ejaz, W. Reconfigurable wireless networks. Proc. IEEE 2015, 103, 1125-1158. [CrossRef]

29. Zeng, Y.; Lyu, J.; Zhang, R. Cellular-Connected UAV: Potential, Challenges, and Promising Technologies. IEEE Wirel. Commun. 2019, 26, 120-127. [CrossRef]

30. Saleem, Y.; Rehmani, M.H.; Zeadally, S. Integration of cognitive radio technology with unmanned aerial vehicles: Issues, opportunities, and future research challenges. J. Netw. Comput. Appl. 2015, 50, 15-31. [CrossRef]

31. Jacob, P.; Sirigina, R.P.; Madhukumar, A.; Prasad, V.A. Cognitive radio for aeronautical communications: A survey. IEEE Access 2016, 4, 3417-3443. [CrossRef]

32. Huo, Y.; Dong, X. Millimeter-wave for unmanned aerial vehicles networks: Enabling multi-beam multi-stream communications. arXiv 2018, arXiv:1810.06923.

33. Amadeo, M.; Campolo, C.; Quevedo, J.; Corujo, D.; Molinaro, A.; Iera, A.; Aguiar, R.L.; Vasilakos, A.V. Information-centric networking for the internet of things: Challenges and opportunities. IEEE Netw. 2016, 30, 92-100. [CrossRef]

34. Ahlgren, B.; Dannewitz, C.; Imbrenda, C.; Kutscher, D.; Ohlman, B. A survey of information-centric networking. IEEE Commun. Mag. 2012, 50, 26-36. [CrossRef]

35. Jin, Y.; Tan, X.; Feng, W.; Lv, J.; Tuerxun, A.; Wang, K. MANET for Disaster Relief based on NDN. In Proceedings of the 2018 1st IEEE International Conference on Hot Information-Centric Networking (HotICN), Shenzhen, China, 15-17 August 2018; pp. 147-153. 
36. Hasegawa, T.; Tara, Y.; Ryu, K.; Koizumi, Y. Emergency message delivery mechanism in NDN networks. In Proceedings of the 3rd ACM Conference on Information-Centric Networking, Kyoto, Japan, 26-28 September 2016; pp. 199-200.

37. Khan, O.A.; Shah, M.A.; Din, I.U.; Kim, B.S.; Khattak, H.A.; Rodrigues, J.J.; Farman, H.; Jan, B. Leveraging named data networking for fragmented networks in smart metropolitan cities. IEEE Access 2018, 6, 75899-75911. [CrossRef]

38. Barka, E.; Abdelaziz Kerrache, C.; Hussain, R.; Lagraa, N.; Lakas, A.; Hussain Bouk, S. A Trusted Lightweight Communication Strategy for Flying Named Data Networking. Sensors 2018, 18. [CrossRef] [PubMed]

39. Miyano, K.; Shinkuma, R.; Mandayam, N.B.; Sato, T.; Oki, E. Utility based scheduling for multi-UAV search systems in disaster-hit areas. IEEE Access 2019, 7, 26810-26820. [CrossRef]

40. Erim, O.; Wright, C. Optimized mobility models for disaster recovery using UAVs. In Proceedings of the IEEE 28th Annual International Symposium on Personal, Indoor, and Mobile Radio Communications (PIMRC), Montreal, QC, Canada, 8-13 October 2017; pp. 1-5.

41. Katayama, K.; Takahashi, H.; Yokota, N.; Sugiyasu, K.; Kinoshita, T. Cooperation scheme of multi-UAVs for evacuation guidance support. In Proceedings of the IEEE 7th Global Conference on Consumer Electronics (GCCE), Nara, Japan, 9-12 October 2018; pp. 221-222.

42. Erman, A.T.; van Hoesel, L.; Havinga, P.; Wu, J. Enabling mobility in heterogeneous wireless sensor networks cooperating with UAVs for mission-critical management. IEEE Wirel. Commun. 2008, 15, 38-46. [CrossRef]

43. Ezequiel, C.A.F.; Cua, M.; Libatique, N.C.; Tangonan, G.L.; Alampay, R.; Labuguen, R.T.; Favila, C.M.; Honrado, J.L.E.; Canos, V.; Devaney, C.; et al. UAV aerial imaging applications for post-disaster assessment, environmental management and infrastructure development. In Proceedings of the International Conference on Unmanned Aircraft Systems (ICUAS), Orlando, FL, USA, 27-30 May 2014; pp. 274-283.

44. Lim, G.J.; Kim, S.; Cho, J.; Gong, Y.; Khodaei, A. Multi-UAV pre-positioning and routing for power network damage assessment. IEEE Trans. Smart Grid 2018, 9, 3643-3651. [CrossRef]

45. Yamazaki, F.; Kubo, K.; Tanabe, R.; Liu, W. Damage assessment and 3d modeling by UAV flights after the 2016 Kumamoto, Japan earthquake. In Proceedings of the IEEE International Geoscience and Remote Sensing Symposium (IGARSS), Fort Worth, TX, USA, 23-28 July 2017; pp. 3182-3185.

46. Wang, Z.; Wu, L.; Wang, C.; Wen, X.; Niu, Y.; Yin, D. Coactive design of human-machine collaborative damage assessment using UAV images and decision trees. In Proceedings of the Chinese Automation Congress (CAC), Xi'an, China, 30 November-2 December 2018; pp. 1140-1145.

47. Rokhmana, C.A.; Andaru, R. Utilizing UAV-based mapping in post disaster volcano eruption. In Proceedings of the 2016 6th International Annual Engineering Seminar (InAES), Yogyakarta, Indonesia, 1-3 August 2016; pp. 202-205.

48. Dayananda, K.R.; Gomes, R.; Straub, J. An interconnected architecture for an emergency medical response unmanned aerial system. In Proceedings of the IEEE/AIAA 36th Digital Avionics Systems Conference (DASC), St. Petersburg, FL, USA, 17-21 September 2017; pp. 1-6.

49. Cho, D.K.; Chang, C.W.; Tsai, M.H.; Gerla, M. Networked medical monitoring in the battlefield. In Proceedings of the IEEE Military Communications Conference (MILCOM), San Diego, CA, USA, 16-19 November 2008; pp. 1-7.

50. Lagazo, D.; dela Cruz, J.; Solpico, D.; Honrado, J.; Abrajano, G.; Libatique, N.; Tangonan, G. Resilient communications and information systems for disaster-preparedness using UAVs, beacons and data aggregators, via delay-tolerant networks on sub-GHz frequencies. In Proceedings of the IEEE Region 10 Conference TENCON, Jeju Island, Korea, 28-31 October 2018; pp. 1290-1294.

51. Michel, A.; Gettinger, D. Drone Incidents: A Survey of Legal Cases; Bard College Center for Study of the Drone: Annandale-on-the-Hudson, NY, USA, 2017.

52. Alarming Incidents between Drones and Aircraft Have Tripled in Three Years, Says Transport Canada. Available online: https:/ /www.thestar.com/edmonton/2018/08/02/alarming-incidents-between-dronesand-aircraft-have-tripled-in-three-years-says-transport-canada.html (accessed on 6 June 2019).

53. Aljehani, M.; Inoue, M. Performance evaluation of multi-UAV system in post-disaster application: Validated by HITL simulator. IEEE Access 2019, 7, 64386-64400. [CrossRef]

54. Athanasis, N.; Themistocleous, M.; Kalabokidis, K. Wildfire prevention in the era of big data. In Proceedings of the European, Mediterranean, and Middle Eastern Conference on Information Systems, Coimbra, Portugal, 7-8 September 2017; pp. 111-118. 
55. Srivas, T.; Artés, T.; De Callafon, R.A.; Altintas, I. Wildfire spread prediction and assimilation for FARSITE using ensemble Kalman filtering. Procedia Comput. Sci. 2016, 80, 897-908. [CrossRef]

56. Hodgkinson, D.; Johnston, R. Aviation Law and Drones: Unmanned Aircraft and the Future of Aviation; Routledge: Abingdon, UK, 2018.

57. Saxena, D.; Raychoudhury, V.; Suri, N.; Becker, C.; Cao, J. Named data networking: A survey. Comput. Sci. Rev. 2016, 19, 15-55. [CrossRef]

58. Xylomenos, G.; Ververidis, C.N.; Siris, V.A.; Fotiou, N.; Tsilopoulos, C.; Vasilakos, X.; Katsaros, K.V.; Polyzos, G.C. A survey of information-centric networking research. IEEE Commun. Surv. Tutor. 2013, 16, 1024-1049. [CrossRef]

59. Yaqub, M.A.; Ahmed, S.H.; Bouk, S.H.; Kim, D. Interest forwarding in vehicular information centric networks: A survey. In Proceedings of the 31st Annual ACM Symposium on Applied Computing, Pisa, Italy, 4-8 April 2016; pp. 724-729.

60. Ahmed, S.H.; Kim, D. Named data networking-based smart home. ICT Express 2016, 2, 130-134. [CrossRef]

61. Majeed, M.F.; Ahmed, S.H.; Dailey, M.N. Enabling push-based critical data forwarding in vehicular named data networks. IEEE Commun. Lett. 2017, 21, 873-876. [CrossRef]

62. Amadeo, M.; Campolo, C.; Molinaro, A. Internet of things via named data networking: The support of push traffic. In Proceedings of the 2014 International Conference and Workshop on the Network of the Future (NOF), Paris, France, 3-5 December 2014; pp. 1-5.

(C) 2019 by the authors. Licensee MDPI, Basel, Switzerland. This article is an open access article distributed under the terms and conditions of the Creative Commons Attribution (CC BY) license (http://creativecommons.org/licenses/by/4.0/). 